\title{
MASTERPLANNING FOR URBAN CHANGE: A SMALL WORLD METAPHOR
}

\author{
M. GANIS, J. MINNERY \& D. MATEO-BABIANO \\ The University of Queensland, School of Geography, Planning and Environmental Management, Australia.
}

\begin{abstract}
The intent of a masterplanning process is to enable and expedite urban change but sometimes this process is fraught with dissatisfaction. This paper examines the perceptual and conceptual aspects of the masterplanning process and asks (a) What urban qualities make a good place? (b) What are the priorities of a conceptual masterplanning process? and (c) What are the short-term and long-term priorities of a contextual masterplanning process? The participants' results show that urban density and connectivity are the most important aspects of masterplanning places and that stakeholders need an urban change process to enable certainty in the short term and adaptability in the long term. It is proposed that small world network models offer a self-organizing framework for masterplanning short-term and long-term urban change.

Keywords: masterplanning process, small world network metaphor, self-organizing, adaptable, South East Queensland, place making.
\end{abstract}

\section{INTRODUCTION}

Rapid urban change is sometimes fraught with difficult planning negotiations and user dissatisfaction. Masterplanning is intended to diminish anxiety by creating a sense of predictability and inclusion. And yet, the outcomes of well-intentioned, inclusive and rigorous masterplanning processes still seem to startle stakeholders. This paper seeks to understand the masterplanning process as experienced by the key participant groups.

An investigation into the masterplanning process triggers the following questions: What is the stakeholders' perception of their place? What are the priorities of the stages of a masterplanning process? How does the masterplanning timeframe and place context influence these prioritized stages? The study area is South East Queensland, Australia, which is dominated by the City of Brisbane. The popular tourist regions of the Sunshine Coast and the Gold Coast attract development to the north and the south, respectively, and new communities are developing to the west of Brisbane.

South East Queensland is one of the fastest growing regions in Australia and has had a consistent migration of people from other Australian states over many years. Currently, the accommodation of this population drives a long-term urban development and planning process and statutory masterplans are seen as the appropriate vehicle to direct future urban development. However, despite what appears to be a thorough and inclusive process that typically consists of several community consultation meetings; a process of review; public notification and amendment of plans; and finally, adoption of the masterplan by the local government authority, the outcome sometimes sparks community dissatisfaction that belies the rigour of the masterplanning process. This research seeks to dissect the masterplanning process and to offer a new direction in the approach towards planning for urban change.

\section{A SMALL WORLD METAPHOR}

The research proposes a dynamic model that may guide a masterplanning process. Small world network models offer self-organizing characteristics that are dynamic and are time-sensitive $[1,2]$. The alignment between the small world dynamic and urban change processes has been flagged in planning literature [3] but so far, small world theory has been the domain of mainly abstract research in disciplines as diverse as neural, electrical, social and computer network modelling. This present 
research seeks small world characteristics via an empirical study of the masterplanning process to validate small world models as a relevant metaphor for masterplanning in a dynamic urban context.

A well-known idea of the dynamic of urban spatial patterning is space syntax $[4,5]$. Space syntax was developed over two decades ago and for its time ambitiously addressed the built form and social nexus. It was one of the few quantitative attempts to analyse urban space and how people use it. Controversy arises because the interpretation of the quantitative results is often subjective $[6,7]$. Furthermore, the process is limited to a binary approach [8] rather than a multidimensional approach which is likely to be a more appropriate way to analyse complex urban places.

Alternatively, systems thinking offers a theoretical approach to analyse urban complexity because it acknowledges the vicissitudes of order and chaos, certainty and uncertainty [9]. A key issue raised by systems thinkers in planning is how to address the evolution of urban developments. The dimension of time is a dilemma in a planning process because of short-term and long-term aspirations and needs of stakeholders. Systems thinking not only offers some short-term certainty through closed systems, traditional static networks but also addresses long-term uncertainty through feedback loops. However, complexity is a step further towards chaos. The almost chaotic nature of complexity shifts planning into a world that is constantly evolving. The question that confronts planning is this: Should places be planned to come into being or should places be always becoming [9]? The issue of 'being' and 'becoming' is the difference between traditional static network theory (i.e. being a place) and dynamic small world network theory (i.e. becoming a place). But first, what is a small world?

The notion of small world networks stems from Milgram's seminal observation of social networks that coined the notion of 'six degrees of separation' $[10,11]$. Degrees are the connections across social groups that expedite our social affiliations purportedly an average of six affiliates between any two people on the planet - this is the small world.

Milgram's social contacts are typically random and long ranged but they also enable shortcuts between people, groups, neighbourhoods, towns and so on. It is these shortcuts that afford efficient connectivity. Consider the social development of a neighbourhood: neighbourhood social groups usually develop initially via contact with the nearest neighbours [12]. Such social connections are relatively predictable because they are nearby. In time, social contact is made with more distant neighbours and neighbourhoods through work colleagues, interest groups, friends and relatives that make up the many layers of interaction that we cultivate over time. Distance makes social contact between neighbourhoods less predictable and is usually the result of the random contact with a mutual acquaintance prompting the exclamation, 'Small world isn't it!' This truism sparked research into a formal understanding of the self-organizing nature of small world networks.

To begin, imagine living in a caveman world (Fig. 1). In each cave lives a group of cavemen who only know each other and no one else. One curious caveman ventures out and meets another caveman passing by: 'their propensity to be acquainted immediately becomes very high and stays that way regardless of how many additional mutual friends they may have' [13]. Now that our caveman world is connected imagine going to a social gathering during which one cavemen randomly meets another and on discovery of a mutual acquaintance exclaims, 'Small world isn't it!' This mutual acquaintance is a random, long-range shortcut between formerly distant troupes of cavemen. Affiliations via such shortcut acquaintances beyond one's own cave significantly increase our caveman's global connectivity and communication.

A brief outline of network characteristics may explain the relevance of a small world model to a masterplanning process. Traditionally, networks are either regular or random. In an urban context, a regular network such as an orthogonal street grid is coherent because it is ordered, predictable and static. Conversely, a random network as found in a medieval town can be incoherent because it is chaotic, flexible and dynamic. Each of these traditional network typologies has their advantages and 
disadvantages: regular networks are coherent but change slowly if at all; random networks are incoherent but change rapidly.

Small world networks are the middle ground between the extremes of regular and random networks $[1,2,13,14]$. Small world networks differ from the traditional typology of regular and random networks because they include the self-organizing factors of time and growth. This means that small world networks embody the coherent order of regular networks as well as the dynamic flexibility of random ones (Fig. 2). Small world networks self organize over time into dense clusters of nodes with long-range shortcuts that efficiently connect different clusters of nodes that would otherwise be far apart.

In an urban context, network theory may be applied in a simplistic explanation of street patterns. Regular networks are generally typical of western city street grids: they are characteristically coherent grids that are bounded, complete and distributed; streets join and meet. This type of street grid is ordered into a regular symmetrical lattice. Although regular networks have the advantage of coherence, its order is slow to change and random changes tend to disrupt its integrity. An example of a

1

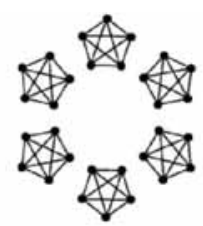

3

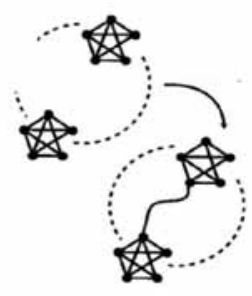

2

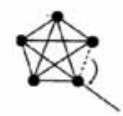

4

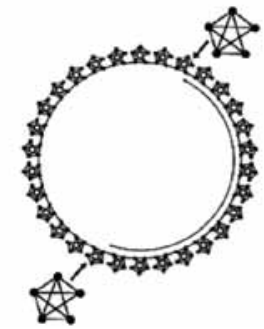

Figure 1: The connected caveman world [14].
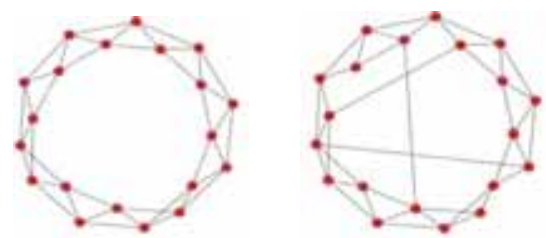

WS small world model Watts and Strogatz (1998)
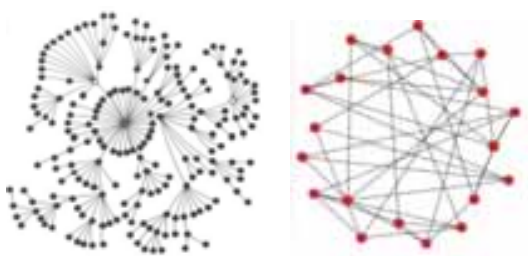

BA scale free model Barabasi and Albert (1999)

REGULAR

SMALL WORLD (2 types)

RANDOM

Figure 2: Network topology [1, 2]. 


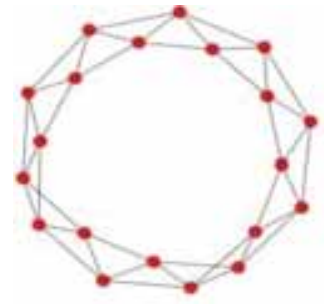

REGULAR NETWORK

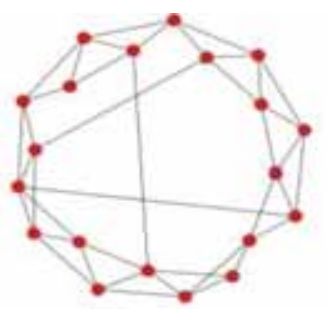

SMALL WORLD NETWORK

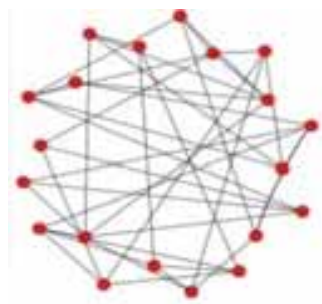

RANDOM NETWORK

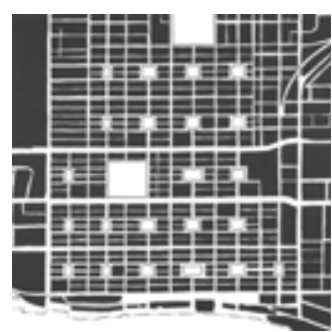

Savannah

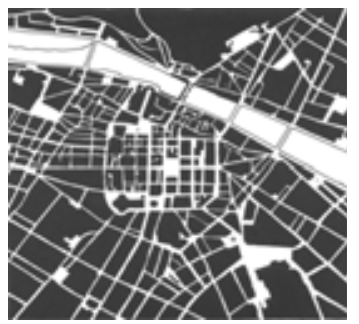

Florence

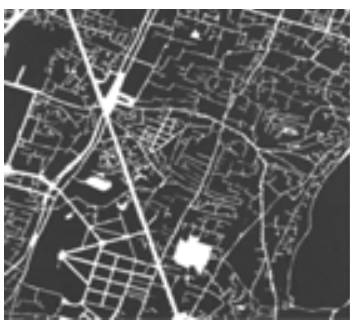

Cairo

Figure 3: A network metaphor for urban street patterns.

regular urban network is Savannah, GA, USA (Fig. 3). Savannah was deliberately planned from a pristine site into an ordered community. Strong egalitarian ideals played a significant role in its initial regular network pattern.

Random networks more typical of eastern city street systems tend to be less coherent: streets may or may not join and meet. This type of network is asymmetrical and tends to be unbounded. Random networks have no fixed pattern and are quick to adapt. The disadvantage of this type of network is its vulnerability to random removal of connections creating an even less coherent urban street network. The largely random street network of Old Cairo, for example, consists of the eroded layers of conquests and caliphs who built and destroyed mosques, military camps and royal compounds around which the haphazard mass of Old Cairo sprawls (Fig. 3). A random change to this street network adapts very quickly to any new connections or disconnections.

The small world of Florence originated as a military camp from which grew an urban network of palatial residences of powerful families, oligarchy, papacy and republicanism fuelled by economic, scientific and cultural predominance (Fig. 3). These palatial residences appear as clustered nodes of powerful families and church domains connected by the fusion of regular and random urban streets. 
The regular street grids of the dense Florentine neighbourhood cluster around squares and the Duomo connected by long-range shortcuts that connect with other more distant places; this is evidence of a random adaptation of the street network to expedite a connection between neighbourhoods. It is not difficult to imagine powerful Florentine families and churches, such as those of the Pitti palace, the Duomo or the Medicis, insisting on a fine pathway from their palace to another to expedite contact with their social milieu.

This present paper uses the self-organizing small world metaphor as a potential masterplanning model because the process that describes the development of a small world network is one that affords urban growth and change. But first, we need to examine the masterplanning process to seek evidence of small world factors. If there is evidence of small world characteristics, it lends support to its potential as a self-organizing model in planning for urban change.

\section{METHOD AND RESULTS}

The research investigated the aspects of place and time in the masterplanning process (Fig. 4). First, the perception of place was investigated and second, the masterplanning process was examined. The place concept mapping task elicited the participants' underlying conceptual structure of their perception of a good place. The conceptual masterplanning task revealed their abstract notions of masterplan priorities and finally, the contextual masterplan task revealed their masterplan priorities in a real context in a Short-Term (ST) and a Long-Term (LT) timeframe. The participants consisted of a stratified random selection of design, development, community professionals and others, living in South East Queensland, Australia. The groups were Architects (10); Councillors (local government elected representatives) (8); Developers (7); Landscape Architects (7); Planners (24); Transport Engineers/Planners (11); and Others (a diverse group) (9). These participant groups were selected because they represent the key negotiators in masterplanning processes in South East Queensland.

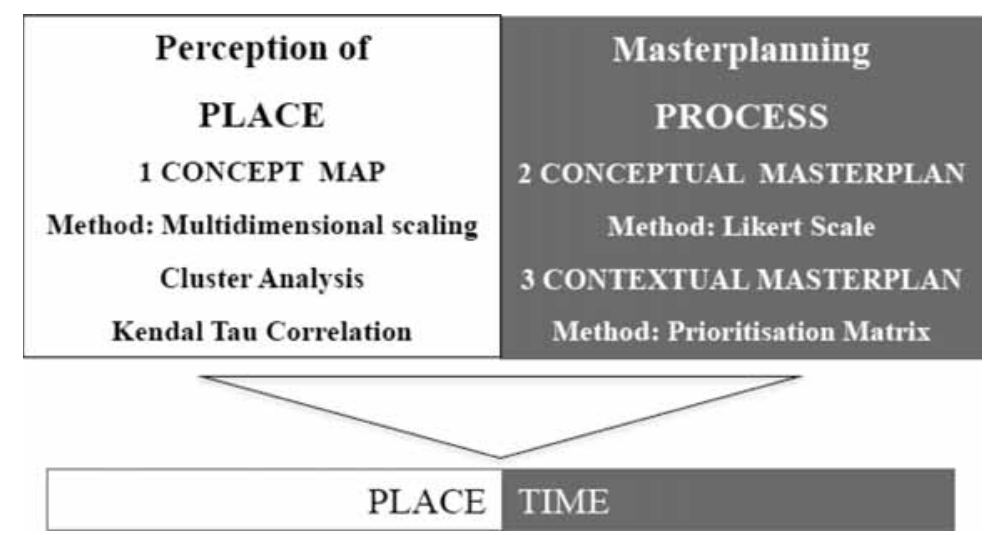

Figure 4: Research plan. (1) The concept map aims at revealing a multidimensional plot of the participants' psychological map of the urban design qualities that make good places. (2) The conceptual masterplanning task aims as revealing the participants' conceptual priorities in a masterplanning process. (3) The contextual masterplanning timeframe task aims at finding out the difference between masterplanning in a ST timeframe and in a LT timeframe and place context. 


\subsection{Concept mapping task: method}

The aim of the concept mapping task was to reveal a psychological map of the participants' perception of what makes a good place. Multidimensional scaling analysis (MDS) was used because it is a non-linear analysis technique that aligns with non-linear small world network theory.

A semantic differential task presented bipolar categories of urban design qualities that were derived from a content analysis of the urban design literature. The participants were asked to rank the following good urban design qualities in South East Queensland (Table 1).

A MDS was used because it is a relational method traditionally used in psychology and social network analysis $[15,16]$. Here, it is used to plot the stakeholders' concept maps to reveal their perception of good urban places by illustrating the network of urban design variables. Cluster Analyses (CA) supported the interpretation of the MDS plots. If there was ambiguity between the MDS and the CA, the MDS result took precedence [17]. The MDS plots were interpreted from the perspective of recent insights into network theory discussed previously.

\subsubsection{Results}

The results of the MDS analyses configure plots consisting of clusters of variables (shown as groups of dots) and dimensions (shown as a line or curve of dots). The results are two-dimensional for clear interpretation. The stress value of an MDS analysis is an evaluation the results' goodness-of-fit: a stress value of $<0.15$ is a 'good fit' and one that is $>0.25$ is a 'bad fit'. The lower stress value indicates greater reliability and the higher the stress value indicates that the results may be too complex to interpret.

These results indicate a range of stress values for each group between $>0.01$ and $<0.16$. This is a 'good fit' not only because the MDS plots are two-dimensional (a better fit is attained with more dimensions) but also the variables are relatively complex concepts open to broad interpretation.

The clusters and dimensions illustrated in the plots indicate the variables that are likely to be conceptually related. As such, those plots that show a more connected network of variables may represent those participants' perceptions that are integrated and those that are more fragmented. Although the MDS results show that each group's plot is different, there is one compelling relationship of variables: the All Groups plot (76 participants) Variable 1 (compact, distinct urban centres); Variable 3 (clear way-finding); and Variable 9 (movement network connectivity).

Table 1: Bipolar categories of urban design quality variables. These variables were derived from a qualitative content analysis of the urban design literature and represent opposite dimensions of urban design qualities of places.

\begin{tabular}{lll}
\hline Var & & Bipolar categories \\
\hline 1 & Compact, distinct centres & Dispersed sub-centres unusual, \\
2 & Local character building style & Landmark architecture \\
3 & Clear way-finding & Mysterious exploration \\
4 & Technical efficiency & Large-scale sensory experience \\
5 & Small-scale local infrastructure & Regional infrastructure \\
6 & Adventurous places & Safe places \\
7 & Urban-rural fusion & Ecological conservation \\
8 & Evolving places & Completed places \\
9 & Movement network connectivity & Movement network separation \\
\hline
\end{tabular}


Table 2: Concept Mapping Task, MDS plots. This is a conceptual map that illustrates the relationship between the urban design quality variables (Table 1). Urban design quality variables that are related are close together and those that are unrelated are far apart. The plots show variables that have a strong relationship as clusters (circled) and dimensions (dotted lines). The plots enable a visual examination of those participant groups who are similar in their perception of place and those who are dissimilar.

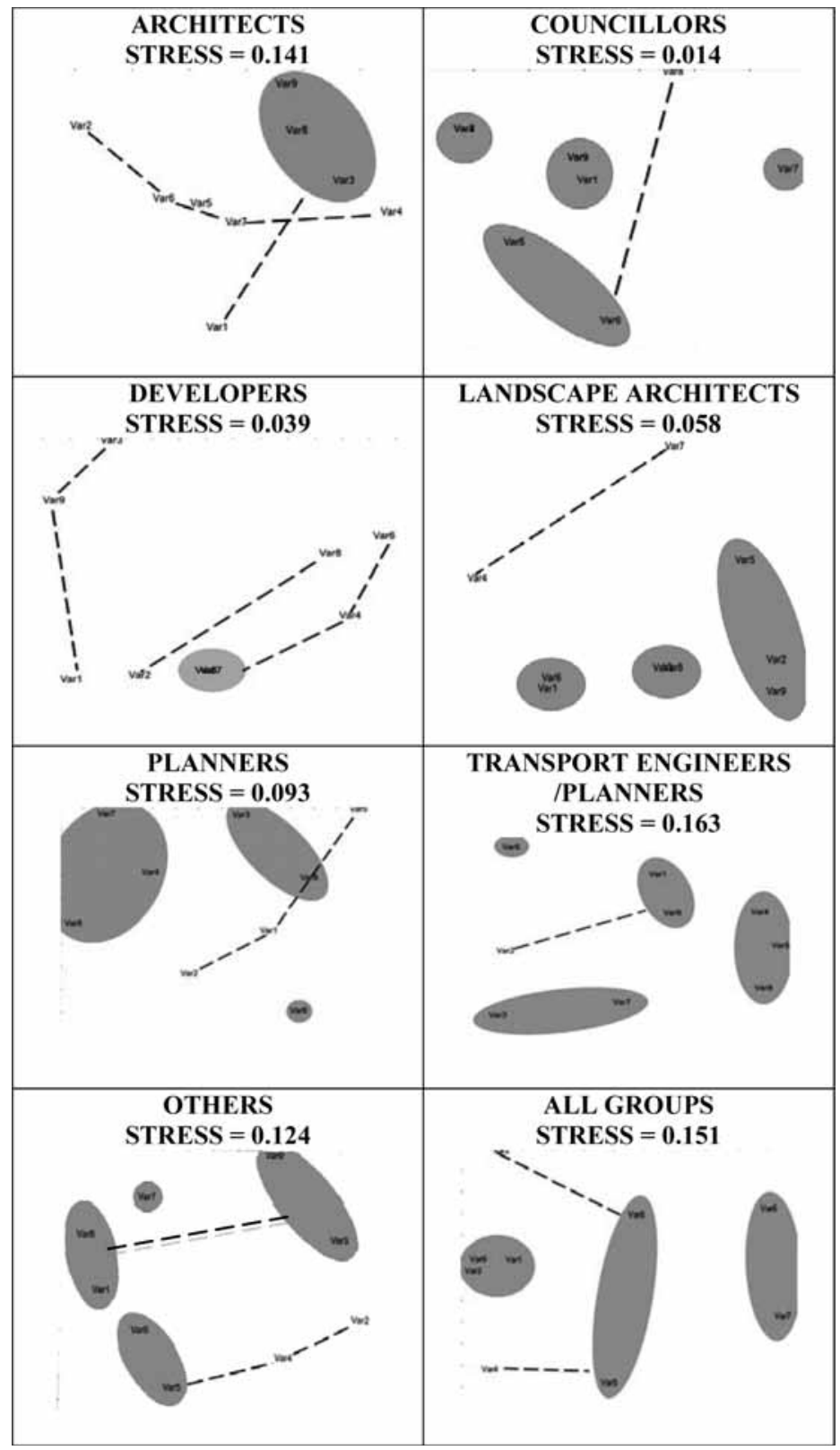


Table 3: Kendall Tau correlations. The significant correlation coefficients (shown in bold) show those groups whose concept map is similar (close to 1) or dissimilar. A: architects; C: councillors; D: developers; LA: landscape architects; P: planners; TE/P: transport engineers/ planners; O: others.

Kendall Tau correlations MD pair-wise deleted marked correlations (shown in bold) are significant at $p<.05000$

\begin{tabular}{lllllllll}
\hline & ALL & A & C & D & LA & P & TE/P & O \\
ALL & 1.000 & $\mathbf{0 . 9 4 4}$ & 0.309 & $\mathbf{0 . 7 4 3}$ & $\mathbf{0 . 7 0 4}$ & $\mathbf{1 . 0 0 0}$ & $\mathbf{0 . 8 1 6}$ & $\mathbf{0 . 8 5 7}$ \\
A & $\mathbf{0 . 9 4 4}$ & 1.000 & 0.253 & $\mathbf{0 . 8 0 0}$ & $\mathbf{0 . 6 4 7}$ & $\mathbf{0 . 9 4 4}$ & $\mathbf{0 . 7 6 0}$ & $\mathbf{0 . 8 0 0}$ \\
C & 0.309 & 0.253 & 1.000 & 0.289 & $\mathbf{0 . 5 1 4}$ & 0.309 & 0.342 & 0.434 \\
D & $\mathbf{0 . 7 4 3}$ & $\mathbf{0 . 8 0 0}$ & 0.289 & 1.000 & $\mathbf{0 . 6 6 6}$ & $\mathbf{0 . 7 4 3}$ & $\mathbf{0 . 5 7 9}$ & $\mathbf{0 . 7 0 5}$ \\
LA & $\mathbf{0 . 7 0 4}$ & $\mathbf{0 . 6 4 7}$ & $\mathbf{0 . 5 1 4}$ & $\mathbf{0 . 6 6 6}$ & 1.000 & $\mathbf{0 . 7 0 4}$ & $\mathbf{0 . 5 1 4}$ & $\mathbf{0 . 7 8 2}$ \\
P & $\mathbf{1 . 0 0 0}$ & $\mathbf{0 . 9 4 4}$ & 0.309 & $\mathbf{0 . 7 4 3}$ & $\mathbf{0 . 7 0 4}$ & 1.000 & $\mathbf{0 . 8 1 6}$ & $\mathbf{0 . 8 5 7}$ \\
TE/P & $\mathbf{0 . 8 1 6}$ & $\mathbf{0 . 7 6 0}$ & 0.342 & $\mathbf{0 . 5 7 9}$ & $\mathbf{0 . 5 1 4}$ & $\mathbf{0 . 8 1 6}$ & 1.000 & $\mathbf{0 . 7 2 4}$ \\
O & $\mathbf{0 . 8 5 7}$ & $\mathbf{0 . 8 0 0}$ & 0.434 & $\mathbf{0 . 7 0 5}$ & $\mathbf{0 . 7 8 2}$ & $\mathbf{0 . 8 5 7}$ & $\mathbf{0 . 7 2 4}$ & 1.000 \\
\hline
\end{tabular}

A Kendall Tau correlation analysis (Table 3) reveals correlation coefficients indicating those groups that have similar concept maps and those that do not. Briefly, a coefficient that is nearest 1 indicates the groups that are most closely related (a perfect correlation being 1) in their conceptual structure of good urban places, and those furthest from 1 less related.

The results show that the only group that has some correlation with the Councillors $(\mathrm{C})$ is the group of Landscape Architects (LA) with a coefficient of 0.5, which may be considered somewhat borderline. The groups with a coefficient indicating similar perceptions are the Architects (A) and the Planners $(\mathrm{P})$ with a coefficient of 0.94 . The groups that are least correlated are Councillors $(\mathrm{C})$ and Architects (A) with a coefficient of 0.25 and Planners (P) with a coefficient of 0.31 .

\subsection{Conceptual masterplanning: method}

The aim of the conceptual masterplanning question was to elicit the participants' abstract notion of the priorities in the stages of a masterplanning process. The participants ranked each masterplanning priority from 1 (unimportant) to 5 (extremely important) on a Likert Scale. The stages presented were derived from the urban design compendium [18] (Table 4).

\subsubsection{Results}

The results show that traffic and pedestrian network is the most important priority followed closely by the site and context analysis and vision and creative ideas. The importance of traffic and pedestrian network appears as a fundamental aspect of the masterplanning process. This insight offers a key direction in the examination of masterplanning in that the small world dynamic of connectivity is revealed as a core urban process.

Moderately important priorities are those that tend to guide a masterplanning outcome: uses and activities; streetscape design and landscaping; urban design principles; and the technical reports. The level of importance of these suggests that the participants' priorities appear to have shifted from the overall visionary concept to the aspects that guide the structure of a masterplan. 
Table 4: Conceptual masterplanning priorities. These masterplanning stages and priorities are conceptual because they are not related to a time or place context.

\section{Technical preparation}

Technical reports (mean 4.18; std. dev. 0.79); community consultation (mean 4.00; std. dev. 0.97)

\section{Design preparation}

Site and context analysis (mean 4.51; std. dev. 0.69); urban design principles (mean 4.20; std. dev. 0.76); vision and creative ideas (mean 4.49; std. dev. 0.60)

\section{Designing}

Uses and activities (mean 4.33; std. dev. 0.73); traffic and pedestrian network (mean 4.61; std. dev. 0.52); streetscape design and landscaping (mean 4.28; std. dev. 0.75); building form, height and mass (mean 4.04; std. dev. 0.82); architectural character (mean 3.78; std. dev. 0.87)

\section{Monitoring}

Urban design quality appraisal (mean 3.82; std. dev. 0.89); design review and masterplan updating (mean 4.03; std. dev. 0.80)

Table 5: Contextual masterplanning timeframe statements. The conceptual masterplanning stages are retained in this task but the participants are now asked to consider these statements in the context of masterplanning in a ST and LT timeframe and place.

STATEMENT: "Within the timeframe, this stage of the masterplanning process should demonstrate..."

Relevance to the community, certainty for the development industry, delivery of the outcome on time and within budget, flexibility and adaptability, certainty for the community, a detailed urban design/architectural vision, fulfilment of technical growth predictions, environmental and social justice

Finally, those aspects of lesser importance are: building form, height and mass; design review and masterplan updating; community consultation; urban design quality appraisal; and architectural character. Interestingly, community consultation appears in this group of lesser priority which is counter intuitive. Usually, community consultation is considered of great importance at the initial stages of a masterplanning process.

\subsection{Contextual masterplanning timeframe: method}

The aim of the contextual masterplanning timeframe task was to insert the factors of time and place into masterplanning process. The aim was to elicit any differences between masterplanning for the ST and for the LT. The participants completed a prioritization matrix consisting of: technical preparation; design preparation; designing; and monitoring. The context is described as a typical urban centre in South East Queensland $\left(2 \mathrm{~km}^{2}\right)$ within a ST development timeframe (ST $=5-10$ years) and a LT timeframe (LT $=20-25$ years $)$. A score out of $10(1=$ low to $10=$ high $)$ indicates a level of agreement (Table 5).

\subsubsection{Results}

A Wilcoxon Matched Pairs test reveals the variables that are significantly different between responses given in a ST timeframe and those in a LT timeframe. The $p$ level $<0.05$ indicates a 
significant difference in the variable for each timeframe and within each stage. Frequencies and the standard deviations (std. dev.) are also shown to support the interpretation of the results. The results show that.

Technical Preparation is considered relevant for all stages in both timeframes except for one item that is more important in the ST: Delivery of the outcome on time and within budget (ST mean 8.09 std. dev. 1.51; LT mean $6.93 \mathrm{std}$. dev. 1.84). As an issue of preparation it is plausible that delivery time and cost are critical in the ST.

Design Preparations that are more importance in the ST than in the LT: Relevance to the community (ST mean 7.39 std. dev. 2.09; LT mean 6.94 std. dev. 2.12).

Certainty for the development industry (ST mean 7.35 std. dev. 1.89; LT mean 6.82 std. dev. 2.01).

Delivery of the outcome on time and within budget (ST mean 7.87 std. dev. 1.43; LT mean 7.06 std. dev. 2.05).

A detailed urban design/architectural vision (ST mean 7.62 std. dev. 1.96; LT mean 7.24 std. dev. 1.97).

These statements address the stakeholders' expectations in the preparation and implementation of a masterplan. Issues of relevance, certainty, time and cost and architectural documentation highlight the delivery of expected outcomes more important in a ST timeframe.

After the design preparation stage, the masterplanning process takes on a more immediate intent. The items of concern are those that impact upon certainty and delivery in the ST: Certainty for the development industry (ST mean 7.59 std. dev. 1.79; LT mean 7.19 std. dev. 1.99).

Delivery of the outcome on time and within budget (ST mean 8.16 std. dev. 1.46; LT mean 7.25 std. dev. 2.06).

Certainty for the community (ST mean 7.67 std. dev. 1.54; LT mean 7.14 std. dev. 1.86).

If we compare these designing results with the previous design preparation results, there appears to be a shift from issues concerned with stakeholders' expectations (design preparation) to those concerned with the satisfaction of stakeholders' expectations (designing). This seems plausible as immediate issues of certainty and delivery dominate as an important ST outcome.

The results for monitoring indicate that most issues are important in both timeframes except for one item that is considered more relevant in the ST: Delivery of the outcome on time and within budget (ST mean 7.55 std. dev. 1.83; LT mean 6.98 std. dev. 2.10).

This result parallels the first stage of a masterplanning process (technical preparation). This highlights the importance of time and cost in the technical preparation of a masterplan and in the monitoring of those costs in the ST.

In sum, the results of first, the concept mapping task show the importance of Cluster and Connectivity in stakeholders' perception of place. Second, the conceptual masterplanning stages results confirmed the importance of Connectivity as a masterplanning concept. Finally the masterplanning timeframe results which established a timeframe and urban context to the masterplanning stages revealed that at every stage from technical preparation to monitoring, ST issues were more important priorities than LT issues. The following discussion will discuss how these results indicate that a small world model may be a useful metaphor for a self-organizing masterplanning process.

\section{DISCUSSION}

Small world networks are self-organizing because time and change are inherent factors of these types of networks. A small world represents a network topology that combines the order of a regular network and the flexibility of a random one. The small world dynamic is typically one in which clusters of contiguous nodes form hubs (neighbourhoods) that connect with other hubs via longrange shortcuts. 
Briefly, the key outcomes of the empirical study revealed the small world dynamic of connectivity, stakeholder need for masterplanning certainty in the ST and by implication the need for masterplanning flexibility in the LT. These outcomes are surmised from the tasks that targeted priorities of stakeholders' perception of a good place, the priorities of a conceptual masterplanning process and also a contextual masterplanning process.

\subsection{Concept mapping}

The results of the concept mapping task illustrated the differences between stakeholders' perception of a good urban place. The difference between the groups was confirmed by the Kendall Tau correlation that showed that the Councillors' conceptual structure was generally the polar opposite of most other groups except for the Landscape Architects. Nevertheless, the compelling result of the All Groups Concept Map offered an insight that resounded with all the participants' perception of place and may be useful as a common starting point for discussion in planning and negotiating urban change.

\subsubsection{Cluster and connectivity}

The content of the most compelling cluster of the All Groups' plot was (Fig. 5): Variable 1 compact, distinct centres; Variable 3 clear way-finding; and Variable 9 movement network connectivity, is categorized as cluster and connectivity here. As an urban design concept, this relationship of variables describes a good urban place as one that has density and connectivity. The urban design quality of 'compact, distinct centres' promotes urban density and strong local connections not only of the built form, infrastructure and transportation network, community and professional affiliations but also of the distinctiveness of socio-cultural identity and the nuances of place identity.

In this study, the category 'compact, distinct centres' was opposed by 'dispersed sub-centres' so that the participants had a measure of comparison for their response. Translated into the imagery of
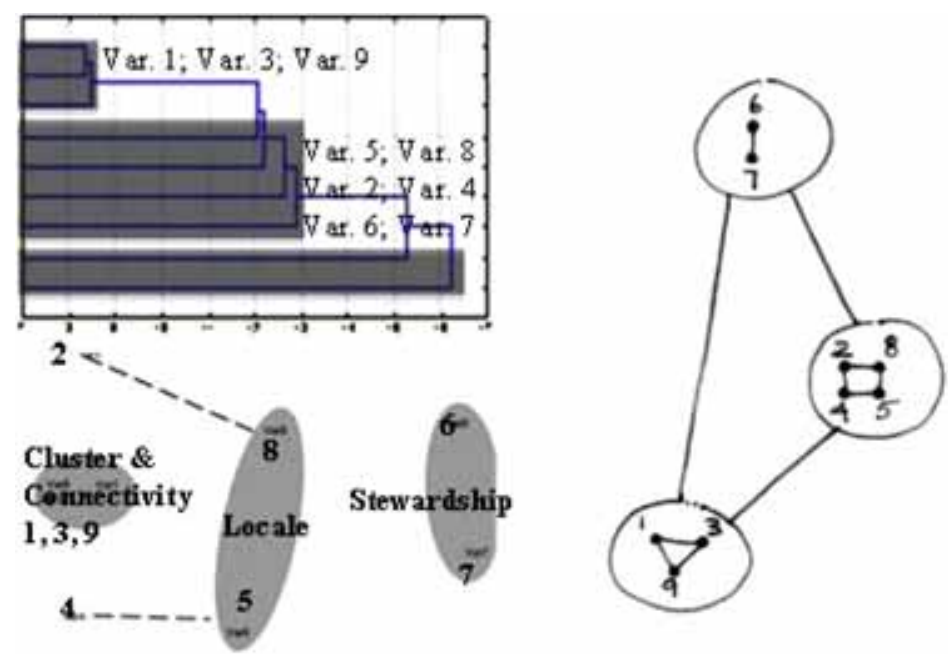

Figure 5: All Groups CA; MDS plot; and small world diagram. The CA (tree diagram) supports the configuration of the MDS plot. This provides a conceptual idea for the clusters and connections for the small world network diagram. 
a real place, 'compact, distinct centres' represented a traditional notion of urban life in which there is a defined town centre that is accessed, recognized, shared and used by a whole community. This may be at varying scales from a local neighbourhood to a village, town, city or distinctive parts of a megalopolis. Conversely, 'dispersed sub-centres' represented sub-urban places that are typically less dense and more scattered with smaller activity centres that are accessible to only a limited community. These places have a greater focus on open space, seclusion and exclusion rather than urban density, population and congregation.

The urban qualities of 'clear way-finding' and 'movement network connectivity' were interrelated as they both connote a transportation system that is easy and efficient rather than convoluted and obscure. 'Clear way-finding' was opposed by 'mysterious exploration'; the former implies a street network that is easy to remember and relatively predictable, whereas the latter suggests a street network that is unpredictable and relatively adventurous or challenging. In this study, the participants preferred an exploratory experience that was efficient and easy rather than convoluted and obscure.

The exploratory experience of 'clear way-finding' was associated with 'movement network connectivity', which implies a network type that expedites the connections between places near and far. 'Movement network connectivity' was opposed by 'movement network separation'. The former implied a transportation system that is integrated with pedestrian, bicycle, private vehicles and mass transport within a street network that is connected throughout. Alternatively, 'movement network separation' implied a transportation system that isolates these varying pathways so that they do not interconnect but are exclusive movement systems - pedestrians only, bicycles only, trucks only and so on - with safety and order as the main driver.

This cluster of urban design variables represented the core cluster of the participants' perception of a good urban place. If we consider the cluster as a whole - 'compact, distinct centres, clear wayfinding and movement network connectivity' - this represents a dense urban centre that consists of an easily accessible hub comprising many and varied uses to which communities flock and feel they belong.

\subsubsection{Locale}

The cluster of variables: Variable 5 Small-scale local infrastructure; and Variable 8 Evolving places; Variable 2 Local character building style; and Variable 4 Sensory experience was locally connected with each other and globally connected with the hub Cluster and Connectivity. This cluster of variables was defined as Locale because these qualities describe the urban setting not only in concrete terms, such as infrastructure and building style, but also in the abstract terms of urban evolution and sensation. This cluster Locale favoured smaller scale local infrastructure and a distinctive architectural style typically associated with traditional urban places and supported the hub, Cluster and Connectivity. An important aspect of this Locale cluster was that of 'evolving places'. This indicated the participants' recognition of a self-organizing process of urban change over that of a finite notion of 'completed places'.

\subsubsection{Stewardship}

Finally, the cluster of urban qualities and the one with the longest global connection with the hub Cluster and Connectivity, but a closer global connection with Locale consisted of: Variable 6 Safe places; and Variable 7 Ecological conservation. This cluster was defined here, as Stewardship because these urban design variables are associated with a sense of responsibility for the safety of people and the environment. As such, Stewardship represented the participants' role and responsibility and indicated the influence of their knowledge, experience and the dispersion of urban design ideas between the stakeholder groups [19]. 


\subsection{Conceptual masterplanning}

The results of the conceptual masterplanning process revealed the participants' conceptual masterplanning priorities. The most important stage was the technical preparation of the priority, traffic and pedestrian network which confirmed the importance of urban connectivity; a key small world dynamic.

The small world factor of time is implied in the next most important priorities: the site and context analysis and the vision and creative ideas. On the one hand, the site and context analysis examines a place in present time and on the other hand the vision and creative ideas explores a place in the future. This means that conceptually at least, the participants had at the outset identified the two key characteristics of a self-organizing small world - time and change - as the most important aspects of a masterplanning process.

The dynamic notion of urban connectivity and the visionary masterplan over time is the setting for the issues of urban structure that were moderately important: uses and activities; streetscape design and landscaping; urban design principles; and the technical reports. Next, the building form, height and mass; and architectural character indicated less important stages of a masterplanning process. Finally, the results showed that the least important masterplanning stages are the monitoring issues of design review and masterplan updating; and urban design quality appraisal. Interestingly, within the group that indicated least importance, there appears to be an anomaly: community consultation.

Community consultation is usually considered as one of the most important parts of the preparatory stages of a masterplanning process and yet these results ranked it at the tail-end stage. Perhaps the lack of a context and timeframe diminished the relevance of community input because in this question the masterplanning process was just an abstract notion. This suggests that at a conceptual masterplanning level, which has neither timeframe nor context, community consultation was an afterthought.

\subsection{Contextual masterplanning timeframe}

This task inserted the factors of time and place context into the masterplanning process. Interestingly, in this task relevance to the community was significant in the ST design preparation stage in contrast to the result in the conceptual masterplanning priorities task previously. Furthermore, certainty for the community was also significant in the ST designing stage. This appears to support the notion that community consultation becomes relevant with the introduction of a real-world context within a ST timeframe.

Once a context and timeframe was introduced, the differences between masterplanning in ST and LT timeframes were illuminated. The results elevated the importance of relevance, certainty and delivery particularly in the ST design preparation and designing stages: almost half the items in these stages were more important in the ST. If relevance, certainty, and delivery are ST imperatives does this mean that uncertain or more flexible aspects of masterplanning are LT imperatives?

Interpreted as planning metaphor, it is proposed here that a small world model offers a masterplanning framework, which delivers certainty and relevance in the ST and accommodates uncertainty, flexibility and adaptability in the LT: ST certainty being a product of order and LT flexibility being a process of adaptation. As discussed previously, a small world model is the middle ground between these extremes and is proposed as a self-organizing, masterplanning model that has the flexibility of randomness and the coherence of order.

The results appeared to support the proposal that masterplans need to offer a LT, self-organizing capacity of randomness within the ST certainty of order. And yet, many masterplans are prepared 
modelling neighbourhoods 20 to 50 years in the future. A small world approach to masterplanning means that masterplans may be structured to facilitate ST certainty via clusters of 'nearest neighbour' connections [12-14] and LT adaptability via long-range shortcuts opportune to a future time and place.

In sum, tenets for a framework for a masterplanning process emerged by combining the key outcomes of the results for (a) the concept mapping task, (b) the conceptual masterplanning task and (c) the contextual masterplanning timeframe task.

The results of the concept mapping task revealed the dynamic structure of a small world network illustrated by the dense local connections and long-range global connections of the All Groups' concept of a good urban place, the hub of which was Cluster and Connectivity.

The importance of connectivity was confirmed by the results of the conceptual stages task, which allocated a high priority to the technical preparation stage of traffic and pedestrian network. Finally, the contextual masterplanning timeframe task revealed the importance of ST certainty in masterplanning and LT adaptability. Based on the integration of these outcomes, a framework for a masterplanning process needs to embody cluster and connectivity, ST certainty and the selforganizing capacity of LT adaptability. It is proposed here that a small world metaphor aligns with these characteristics and may offer a framework for a planning process that is both orderly and serendipitous.

\section{CONCLUSION}

Planning for urban change needs a model embedded in certainty and the self-organizing capacity of adaptability over time. The results of the research indicated that participants identified cluster and connectivity, ST certainty and LT adaptability as tenets for a masterplanning process. These key expectations for a masterplanning process appear to align with the dynamic of a small world network: time and change. The results of this research flag an alignment between small world network theory as a metaphor that may be useful as a framework to structure a self-organizing masterplanning process that offers the order of certainty and the adaptability of randomness.

\section{REFERENCES}

[1] Watts, D.J. \& Strogatz, S., Collective dynamics of 'small world' networks. Nature, 393(4), pp. 440-442, 1998. doi: http://dx.doi.org/10.1038/30918

[2] Barabasi, A.-L. \& Albert, R, Emergence and scaling in random networks. Science, 286, pp. 509-512, 1999. doi: http://dx.doi.org/10.1126/science.286.5439.509

[3] Batty, M., Cities as small worlds. Environment and Planning B: Planning and Design, 28, pp. 637-638, 2001. doi: http://dx.doi.org/10.1068/b2805ed

[4] Hillier, B. \& Hanson, J., The social logic of space. Cambridge University Press: London, 1984. doi: http://dx.doi.org/10.1017/CBO9780511597237

[5] Hillier, B. \& Penn, A., Rejoinder to Carlo Ratti. Environment and Planning B: Planning and Design, 31, pp. 501-511, 2004. doi: http://dx.doi.org/10.1068/b3019a

[6] Ratti, C., Urban texture and space syntax: some inconsistencies. Environment and Planning B: Planning and Design, 31, pp. 1-15, 2004. doi: http://dx.doi.org/10.1068/b3019

[7] Bafna, S., Space syntax: a brief introduction to its logic and analytical techniques, Environment and Behavior, 35, pp. 17-29, 2003. doi: http://dx.doi.org/10.1177/0013916502238863

[8] Osman, K.M. \& Suliman, M. The space syntax methodology: fits and misfits. Architecture and Behaviour, 10(2), pp. 189-204, 1994. 
[9] de Roo, G., Being or becoming? that is the question! confronting complexity with contemporary planning theory. A Planner's Encounter with Complexity, In: G. de Roo \& E.A. Silva, Ashgate: Farnham, pp. 19-40, 2010.

[10] Guare, J., Six Degrees of Separation: a play. Vintage Books: New York, 1990.

[11] Milgram, S., The small world problem. Psychology Today, 2, pp. 60-67, 1967.

[12] Kleinberg, J.M., Navigation in a small world. Nature, 406, pp. 845, 2000. doi: http://dx.doi. org/10.1038/35022643

[13] Watts, D.J. Six Degrees: the science of a connected age. Norton: New York, 2003.

[14] Watts, D.J. Small Worlds: the dynamics of networks between order and randomness. Princeton University Press: Princeton, p. 45, 1999.

[15] Wasserman, S. \& Faust, K., Social Network Analysis: methods and applications. Cambridge University Press: Cambridge, 1994. doi: http://dx.doi.org/10.1017/CBO9780511815478

[16] Scott, J., Social Network Analysis: a handbook. SAGE Publications Inc: London, 2000.

[17] Trochim, W., available at www.socialresearchmethods.net/mapping/mapping.htm. 2009.

[18] English Partnerships. Urban Design Compendium. 2008.

[19] Ganis, M., Minnery, J. \& Mateo-Babiano, D, The small world of stakeholder groups: cascading concepts for urban change. WIT Conference Proceedings: Sustainable Development and Planning. Wessex Institute of Technology Publications: Ashurst Lodge, UK, 2011. 